\title{
Pain after laparascopic bilateral hernioplasty
}

\section{Early results of a prospective randomized double-blind study comparing fibrin versus staples}

\author{
E. Boldo $\cdot$ Andres Armelles $\cdot$ Guillermo Perez de Lucia $\cdot$ Felix Martin \\ Juan Pablo Aracil · Juan Manuel Miralles · David Martinez • \\ Javier Escrig
}

Published online: 13 February 2008

(C) Springer Science+Business Media, LLC 2008

\section{Erratum to: Surg Endosc}

\section{DOI 10.1007/s00464-007-9587-z}

The following coauthors were inadvertently omitted:

Andres Armelles; Consorcio Hospitalario Provincial Castellon, Surgery

Guillermo Perez de Lucia; Consorcio Hospitalario Provincial Castellon, Surgery
Felix Martin; Consorcio Hospitalario Provincial Castellon, Surgery

Juan Pablo Aracil; Consorcio Hospitalario Provincial Castellon, Surgery

Juan Manuel Miralles

David Martinez

Javier Escrig

Springer regrets the error.

The online version of the original article can be found under doi: 10.1007/s00464-007-9587-z

E. Boldo $(\varangle) \cdot$ A. Armelles · G. Perez de Lucia - F. Martin ·

J. P. Aracil · J. M. Miralles · D. Martinez · J. Escrig

Surgery, Consorcio Hospitalario Provincial Castellon, Castellon,

Castellon, Spain

e-mail: eboldo@yahoo.com 